\title{
In and Out of the Hospital: The Hidden Interface of High Fidelity Research Via RFID
}

\author{
Svetlena Taneva and Effie Law \\ Swiss Federal Institute of Technology (ETH Zürich), Zurich, Switzerland \\ \{tanevas, lawl\}@ethz.ch
}

\begin{abstract}
The use of RFID technology in HCI research is emerging. We identify its promising application in the healthcare sector by empowering the process of capturing, extracting and analyzing data, which help understand task patterns underlying human errors and other intriguing phenomena. RFID and video as research tools are compared to identify their strengths and weaknesses. RFID can be cost-effective and powerful, especially when combined with the hospital information system and optionally with video analysis.
\end{abstract}

\section{Introduction}

A major impediment to the advancement of safety science in healthcare usability is that it is very difficult to obtain precise data for the activity under consideration. Observations are incomplete and subjective. Video captures a wealth of data, but is limited to a single point of view. Doing research on medical activity is especially challenging as there is a great number of participants and technologies involved in a medical workflow, all distributed across time and space - thus, the sources of data go beyond the boundaries of an operating room, a ward, a floor, or even a hospital building. Multiple cameras can solve the problem of incompleteness, but at the extremely high cost of having to align different tapes. The implication for safety research is a compromise between scope of analysis and completeness.

Radio Frequency Identification (RFID) is being exploited in tangible computing and ubiquitous computing. Within the healthcare sector RFID is currently utilized as an operations management tool. We argue that RFID data complements video data in a way that accelerates the process of analysis by automating and systematizing most data collection, extraction and analysis. Thus, it alleviates many of the issues described and significantly reduces the total cost of analysis. Further, RFID as a research tool is a comparable alternative to video technology in settings where no recording is allowed or where adverse reactions to its use are expected.

\section{RFID as a Research Tool}

RFID is a real-time wireless identification technology that communicates data by radio waves. Data is encoded on a chip, called an RFID tag. Depending on the practical application, tags can store personal information such as patient's name and blood type, and can be coupled with various sensors that provide contextual data to be stored - e.g. temperature, motion, and orientation. 
Tags are usually attached to or integrated in physical objects such as garments, wristbands, medical devices, and blood sample containers. A tag's unique ID number is associated with a patient, staff or object record stored in the hospital's back-end information system. Data written on the tag can be read automatically, without any user intervention, by readers located throughout a hospital. This allows the automatic tracing of patients, staff, lab samples, and assets. Identification is possible with mobile readers as well (PDAs). In many instances safety is significantly improved through real-time alerting and prevention of medical error [4], and the quality of patient care is enhanced [1]. RFID is implemented in the outpatient care services as well - patient medication intake is monitored through home smart-cabinets, and bleeding is detected and treatment remotely adjusted for home hemodialysis patients.

RFID technology is implemented in many hospitals around the world to seamlessly provide information used to improve healthcare operations. We suggest that data RFID technology provides can be used to alleviate many of the problems introduced by video data analysis - RFID data is accurate, objective, time-stamp ordered, and ubiquitous - in and out of the hospital. RFID data, in combination with video, can significantly reduce the total time-cost of healthcare video research. Additionally, RFID data is an adequate replacement of video for situations where video utilization is not possible. Unlike video, there is no setup-cost associated with RFID in hospitals where it is already implemented for operational purposes.

Data collection with RFID technology is transparent to the participants in the healthcare process and therefore prevents the practical challenges of videotaping adverse reactions and sometimes even bans on videotaping, which hinder systematic data sampling in healthcare settings [5]. A leading obstacle to the use of video is also the loss of confidentiality [6], which is easily addressed with RFID data by hiding identity information from an analyst, where required. In addition, people and asset trajectories are easily identifiable with RFID data, as well as trajectory intersections. The frequency of RFID data acquisition is an adjustable parameter.

Data extraction is a major methodological challenge of video research [6]. Data extraction has an extremely high cost with video analysis - transcription and coding are labor intensive; movement through the tape is sequential and thus it is difficult to instantly search for particular types of events; correlating multiple tapes is cumbersome. Extraction with RFID data is reduced to defining appropriate queries over a database. Sequential and predicated data reviewing is possible, and alignment is a trivial task. The raw data together with the results of queries make rich data sets for analysis. However, database knowledge is required to define the search queries.

Data Analysis with video research provides powerful qualitative analysis material. However, the wealth of qualitative information is hard to quantify and requires subjective analysis. The challenge of video empirical data is to produce generalizable theories. RFID data, on the other hand, strips some of the richness of video data in exchange for a systematic quantification of socio-technical data. Events from the Hospital Information System (HIS), such as patient state alarms, can complement RFID data for a richer analysis. Trends and outliers can be detected through automated analysis methods (visualization tools and queries) and these initial analysis outputs can guide the ethnographic video research in a more selective manner, thereby further reducing the time-cost of analysis. The massive amount of data from a HIS could conceal certain events from an analyst, while at the same time affording the 


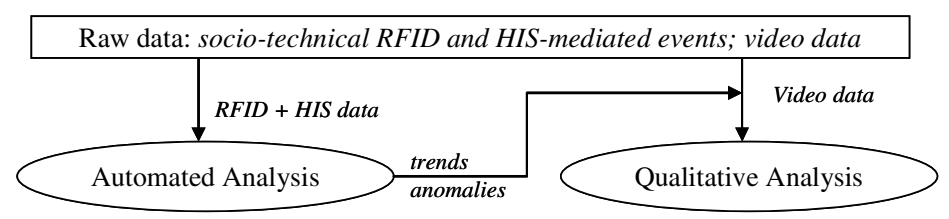

Fig. 1. The process of analysis based on RFID, other HIS data and video data, in its general form. The process is iterative (not shown). Video becomes more of a tool to help explain phenomena (trends, patterns, anomalies) identified through the automated analysis.

advantages of accelerated phenomena discovery [2]. The basic methodology we propose is shown in Fig. 1. Where video data is not available, RFID data can be used to recreate a simulation of the discrete events.

The types of data and steps of analysis that the Exploratory Sequential Data Analysis framework identifies as requirements for solid theory building [3] are mostly covered by data from a HIS with an RFID component. Such data includes all system events; and a subset of the environmental and behavioral events. HIS data is sequential and open to exploratory research. The smoothing operations that guide exploratory video analysis and encourage hypothesis generation are also applicable to RFID and other HIS data analysis (see Table 1).

Table 1. Applying the ESDA smoothing operations [3] is possible with video and RFID data

\begin{tabular}{lll}
\hline \multicolumn{1}{c}{ Smoothing operation } & \multicolumn{1}{c}{ Video } & \multicolumn{1}{c}{ RFID (and HIS) } \\
\hline Chunking data into coherent groups & Manual operation & one-time query definition; automated \\
\hline Commenting data and chunks & Manual & Manual \\
\hline Coding data & Manual operation & Automated \\
\hline Connecting related data elements & Manual operation & Manual and/or automated \\
\hline $\begin{array}{l}\text { Compare data manipulation } \\
\text { outcomes }\end{array}$ & Manual and/or automated & Automated \\
\hline Constrain data views & Manual and/or automated & Automated \\
\hline Convert data views & Manual and/or automated & Automated \\
\hline Compute summary representations & Manual and/or automated & Manual and/or automated \\
\hline
\end{tabular}

Some questions of interest in healthcare safety science are what task patterns lead to human error; what kinds of cooperations emerge in critical situations; and how closely physicians and other staff work together. To answer such crucial questions, one can look at a variety of metrics in the RFID data. We illustrate these in Table 2. Further, one can easily adjust the grain of analysis - from one person's perspective where all system events generated around a person are considered, to a one space perspective - where all events generated within a given physical space are considered.

The automated analysis of RFID data with appropriately defined metrics can reveal rapidly patterns and results that might otherwise be difficult to detect. It is important to look for recurrent patterns and for those situations where the patterns do not hold. Insights from RFID data analysis are powerful guides to the kinds of phenomena that we should look to explain with the more demanding task of video analysis. 
Table 2. Examples of metrics that can be defined over a simple set of RFID data including information on the position and trajectories of patients, staff, and assets. Read error rates are insignificant $(<10 \%)$ and therefore the data provides confident results.

\begin{tabular}{ll}
\hline \multicolumn{1}{c}{ Metric } & \multicolumn{1}{c}{ Operational definition } \\
\hline Interaction between X and Y & The IDs for X and Y are in close proximity for a period of t-time \\
\hline Participation of X in activity & Number of system events from X \\
\hline Degree of cooperation of X with Y & Frequency of close proximity interactions per unit of time \\
\hline Patient seen nurse & Nurse ID and patient ID in close proximity for a period of t-time \\
\hline Patient care quality & $\begin{array}{l}\text { Patient outcomes per illness condition compared with frequency of } \\
\text { "patient seen nurse" and of "length of patient seen nurse" (or } \\
\text { doctor) }\end{array}$ \\
\hline Interruption & $\begin{array}{l}\text { An event causes the nurse to leave the patient within 60secs of the } \\
\text { event. The even can be: another staff comes in close proximity; the } \\
\text { system raises an alarm; the nurse receives a page. }\end{array}$ \\
\hline
\end{tabular}

\section{Concluding Remarks}

The challenges of using video as a research tool for healthcare are high time- and labor-cost, issues of confidentiality, and negative effects on people's behavior. We proposed that in healthcare settings where RFID is implemented, using data from the HIS can relieve most of these issues. The total cost of analysis can be significantly reduced, and the data is objective, accurate, ordered, and ubiquitous. Most of the analysis can be automated to quickly reveal patterns and results. Consequently, it is possible to more selectively engage in the labor-intensive task of video analysis. However, the benefits of using RFID for analysis need to be scientifically validated.

One consideration with our proposed approach is to find an effective way to deal with the massive amounts of data from RFID and other HIS components. Before any analytical processes can begin, data acquisition intervals should be considered, and data filtering requirements appropriate to the research topic need to be established. Also, good database skills are required to define complex and correct queries. In either case, the support of technically knowledgeable individuals is necessary.

\section{References}

1. Bacheldor, B.: Memorial Health OR Unit Chooses UWB RFID. RFID J. (January 16, 2007)

2. Ritter, F.E., Larkin, J.H.: Developing Process Models as Summaries of HCI Action Sequences. Human Computer Interaction 9, 345-383 (1994)

3. Sanderson, P.M., Fisher, C.: Exploratory sequential data analysis: foundations. HumanComputer Interaction 9, 251-317 (1994)

4. Swoboda, S.M., Earsing, K., Strauss, K., Lane, S., Lipset, P.A.: Electronic monitoring and voice prompts improve hand hygiene and decrease nosocomial infections in an intermediate care unit. Crit. Care Med. 2(32), 358-363 (2004)

5. Xiao, Y.: The LOTAS Group. Understanding Coordination in a Dynamic Medical Environment: Methods and Results. In: McNeese, M., Salas, E., Endsley, M. (eds.) New Trends in Collaborative Activities. Hum. Fact. Erg. Society, pp. 242-258. California (2001)

6. Xiao, Y., Mackenzie, C.F.: Introduction to the special issue on Video-based research in high risk settings: Methodology and experience. Cogn. Tech. Work 6, 127-130 (2004) 